\title{
Guest editorial of the IJCARS-BVM 2018 special issue
}

\author{
Andreas Maier ${ }^{1} \cdot$ Thomas M. Deserno $^{2}$ (D) Heinz Handels ${ }^{3} \cdot$ Klaus Maier-Hein ${ }^{4} \cdot$ Christoph Palm $^{5}$. \\ Thomas Tolxdorff 6
}

Published online: 7 January 2019

(c) CARS 2019

It is a great pleasure to present this special issue in the International Journal of Computer Assisted Radiology and Surgery on the German workshop on medical image computing "Bildverarbeitung für die Medizin" (BVM) 2018. After almost 5 years of absence of BVM special issues in IJCARS, we restore this long-standing tradition of hosting the best papers of the conference in this well-reputed journal.

Significant progress is currently being made in the computer-aided processing and automatic analysis of medical image data, and the limits of feasibility are being expanded every day. Since the advent of deep learning, many break-throughs were achieved and new results emerge in rapid succession. For this reason, BVM continues to provide a forum for the presentation and discussion of the latest algorithms, systems, and applications in this field. The aim is to deepen the interaction between scientists, industry, and users as well as the explicit inclusion of young scientists who report on their bachelor, master, doctoral and habilitation projects. BVM very successfully held previous workshops in Aachen,

\footnotetext{
Andreas Maier

andreas.maier@fau.de

Thomas M. Deserno

thomas.deserno@plri.de

Heinz Handels

handels@imi.uni-luebeck.de

Klaus Maier-Hein

k.maier-hein@dkfz-heidelberg.de

Christoph Palm

christoph.palm@oth-regensburg.de

Thomas Tolxdorff

thomas.tolxdorff@charite.de

1 Department of Computer Science, Chair of Computer Science

5 (Pattern Recognition), Friedrich-Alexander-University

Erlangen-Nuremberg, Martensstr. 3, 91058 Erlangen,

Germany

2 Peter L. Reichertz Institute for Medical Informatics, TU Braunschweig and Hannover Medical School, Mühlenpfordtstr. 23, 38106 Brunswick, Germany

3 Institute of Medical Informatics, University of Lübeck, Ratzeburger Allee 160, 23538 Lübeck, Germany
}

Berlin, Erlangen, Freiburg, Hamburg, Heidelberg, Leipzig, Lübeck, and Munich and became a central interdisciplinary forum for the German medical image computing community.

In 2018, BVM returned to Erlangen after 15 years. We could welcome many participants from different countries and had an excellent panel of invited speakers from all over Europe. During the preparation of the conference, a total of 84 submissions, including 60 original works and 24 abstracts underwent a critical review process. As a result, 28 talks, 44 posters, and six software demonstrations were accepted for presentation. Out of these, the best 20 accepted original works were invited to expand their conference paper to be included into this special edition. Fourteen of them actually submitted and after another round of rigorous reviews and major revisions, six of these papers were accepted in time for this special issue. Five out of these six papers address topics of machine or deep learning in medical imaging which is in line with the growing importance of machine learning and pattern recognition in our field.

Aubreville et al. presented a method to automatically detect motion artifacts in confocal laser endomicroscopic images. With their method, they intend to support interventional application of this novel imaging modality and close to real-time in vivo diagnosis of malignancy of the oral epithelium. They report high accuracies of up to $94.8 \%$.

In "Traditional Machine Learning for Limited Angle Tomography," Huang et al. compared linear regression, multilayer perceptrons, and decision forests for reduction of limited angle artifacts in computed tomography. Although their method is not deep but using handcrafted features,

4 Division of Medical Image Computing, German Cancer Research Center, Heidelberg (DKFZ), Im Neuenheimer Feld 581, 69120 Heidelberg, Germany

5 Regensburg Medical Image Computing (ReMIC), Ostbayerische Technische Hochschule Regensburg (OTH Regensburg), Galgenbergstr. 32, 93053 Regensburg, Germany

6 Institute of Medical Informatics, Charité-Universitätsmedizin Berlin, Hindenburgdamm 30, 12200 Berlin, Germany 
they are able to reduce the root-mean-square error between artifact-free and artifact-removed reconstruction down to 29 $\mathrm{HU}$ in interventional C-arm CT geometries.

Bopp et al. proposed further improvements on X-ray phase contrast imaging using dual gratings. In their simulations, they demonstrate that such dual gratings are able to replace the highly absorbing grating G2 directly in front of the detector. As such, low visibilities between 4.5 and $9 \%$ are acceptable as the method allows to reduce the potential patient dose substantially.

In "A Machine Learning Pipeline for Internal Anatomical Landmark Embedding based on a Patient Surface Model," Zhong et al. presented a machine learning method to augment a virtual patient surface model with additional internal landmarks that locate main skeletal bones in the body without additional ionizing radiation using a surface camera only. The average landmark error on 50 landmarks is about $1.2 \mathrm{~cm}$.

Blendowski et al. presented a method to combine Markov Random Fields (MRFs) and deep learning to improve large lung motion estimation for COPD patients. In their method, they propose to learn local binary CNN descriptors from patches with landmark retrieval as auxiliary task. In a second step, they combine this with a dense displacement field sampling that is regularized using an MRF. In their results, they demonstrate that this approach can be improved further using a combination with additional handcrafted features.

In "Automatic bone segmentation in whole-body CT image," Klein et al. used the well-known U-net in different variants. In particular, they compare training from $2 \mathrm{D}$ slices, a pseudo-3D approach using orthogonal slices, and an unsupervised pre-training approach. Their results indicate high agreement of the automatic segmentation and ground truth annotations with Dice scores of 0.95 .

From these excellent contributions, we can observe that deep learning is becoming more and more relevant to our field. Yet, traditional machine learning and combinations of deep and traditional methods still demonstrate high —and in some cases even superior-results. Additionally, there are still emerging technologies such as X-ray phase contrast that might be developed further to bring even more gamechanging potential to the field.

As such, we believe that we were able to assemble an exciting special issue and that you will enjoy these papers. 